\title{
Geometry of bracket-generating distributions of step 2 on graded manifolds
}

\author{
Esmaeil Azizpour ${ }^{a^{*}}$ and Dordi Mohammad Ataei ${ }^{\mathrm{a}}$ \\ ${ }^{a}$ Department of Pure Mathematics, Faculty of Mathematical Sciences, University of Guilan, Rasht, Iran \\ *Corresponding author E-mail: eazizpour@guilan.ac.ir
}

\section{Article Info}

Keywords: Graded manifold, Distribution.

2010 AMS: 58A50, $58 A 30$

Received: 18 April 2018

Accepted: 24 September 2018

Available online: 30 September 2018

\begin{abstract}
A $Z_{2}$ - graded analogue of bracket-generating distribution is given. Let $\mathscr{D}$ be a distribution of rank $(p, q)$ on an $(m, n)$-dimensional graded manifold $\mathscr{M}$, we attach to $\mathscr{D}$ a linear map $F$ on $\mathscr{D}$ defined by the Lie bracket of graded vector fields of the sections of $\mathscr{D}$. Then $\mathscr{D}$ is a bracket-generating distribution of step 2 , if and only if $F$ is of constant rank $(m-p, n-q)$ on $\mathscr{M}$.
\end{abstract}

\section{Introduction}

A smooth distribution $D \subset T M$ is said to be bracket-generating if all iterated brackets among its sections generate the whole tangent space to the manifold $M,[1,8] . D$ is a bracket-generating distribution of step 2 if $D^{2}=T M$, where $D^{2}=D+[D, D]$. Bejancu showed that a distribution of rank $k<m=\operatorname{dim} M$ is a bracket-generating distribution of step 2 , if and only if, the curvature of $D$ is of constant rank $m-k$ on $M,[1]$.

In this paper, a $Z_{2}$-graded analogue of bracket-generating distribution of step 2 is given. Some differences arise in the graded case due to the presence of odd generators. Given a distribution $\mathscr{D}$ of rank $(p, q)$ on an $(m, n)$-dimensional graded manifold $\mathscr{M}$, we attach to $\mathscr{D}$ a linear map $F$ on $\mathscr{D}$ defined by the Lie bracket of graded vector fields of the sections of $\mathscr{D}$. Then $\mathscr{D}$ is a bracket-generating distribution of step 2 , if and only if $F$ is of constant rank $(m-p, n-q)$ on $\mathscr{M}$. In particular, if rank $\mathscr{D}(z)=(m-1, n)$, then for the linear map $F=F_{0}+F_{1}$ associated to $\mathscr{D}, F_{0} \neq 0$ and if $\operatorname{rank} \mathscr{D}(z)=(m, n-1)$, then $F_{1} \neq 0$ on $\mathscr{M}$.

\section{Preliminaries}

Let $M$ be a topological space and let $\mathscr{O}_{M}$ be a sheaf of super $\mathbb{R}$-algebras with unity. A graded manifold of dimension $(m, n)$ is a ringed space $\mathscr{M}=\left(M, \mathscr{O}_{M}\right)$ which is locally isomorphic to $\mathbb{R}^{m \mid n}$, (see [6]).

Let $\mathscr{M}$ and $\mathscr{N}$ be graded manifolds. Let $\phi: M \mapsto N$ be a continuous map such that $\phi^{*}: \mathscr{O}_{N} \longrightarrow \mathscr{O}_{M}$ takes $\mathscr{O}_{N}(V)$ into $\mathscr{O}_{M}\left(\phi^{-1}(V)\right)$ for each open set $V \subset N$, then we say that $\Phi=\left(\phi, \phi^{*}\right): \mathscr{M} \longrightarrow \mathscr{N}$ is a morphism between $\mathscr{M}$ and $\mathscr{N}$.

Let $A$ be a super $\mathbb{R}$-algebra, $\varphi \in E n d_{\mathbb{R}} A$ is called a derivation of $A$, if for all $a, b \in A$,

$$
\varphi(a b)=\varphi(a) \cdot b+(-1)^{|\varphi||a|} a \cdot \varphi(b),
$$

where for a homogeneous element $x$ of some graded object, $|x| \in\{0,1\}$ denotes the parity of $x$ (see [6]).

A vector field on $\mathscr{M}$ is a derivation of the sheaf $\mathscr{O}_{M}$. Let $U \subset M$ be an open subset, the $\mathscr{O}_{M}(U)$-super module of derivations of $\mathscr{O}_{M}(U)$ is defined by

$$
T \mathscr{M}(U):=\operatorname{Der}\left(\mathscr{O}_{M}(U)\right) \text {. }
$$

The $\mathscr{O}_{M}$-module $T \mathscr{M}$ is locally free of dimension $(m, n)$ and is called the tangent sheaf of $\mathscr{M}$. A vector field is a section of $T \mathscr{M}$. If $\Omega^{1}(\mathscr{M}):=T^{*} \mathscr{M}$ be the dual of the tangent sheaf of a graded manifold $\mathscr{M}$, then it is the sheaf of super $\mathscr{O}_{M}$-modules and

$$
\Omega^{1}(\mathscr{M}):=\operatorname{Hom}\left(T \mathscr{M}, \mathscr{O}_{M}\right) .
$$


It is called the cotangent sheaf of a graded manifold $\mathscr{M}$, and the sections of $\Omega^{1}(\mathscr{M})$ are called super differential 1-forms [2, 6]. Let $\mathscr{M}=\left(M, \mathscr{O}_{M}\right)$ be an $(m, n)$-dimensional graded manifold and $\mathscr{D}$ be a distribution of rank $(p, q)(p<m, q<n)$ on $\mathscr{M}$. Then for each point $x \in M$ there is an open subset $U$ over which any set of generators $\left\{D_{i}, D \mu \mid 1 \leq i \leq p, 1 \leq \mu \leq q\right\}$ of the module $\mathscr{D}(U)$ can be enlarged to a set



of free generators of $\operatorname{Der} \mathscr{O}_{M}$, [3].

We attach to $\mathscr{D}$ a sequence of distributions defined by,

$$
\mathscr{D} \subset \mathscr{D}^{2} \subset \ldots \subset \mathscr{D}^{r} \subset \ldots \subset \operatorname{Der} \mathscr{O}_{M},
$$

with

$$
\mathscr{D}^{2}=\mathscr{D}+[\mathscr{D}, \mathscr{D}], \ldots, \mathscr{D}^{r+1}=\mathscr{D}^{r}+\left[\mathscr{D}, \mathscr{D}^{r}\right],
$$

where

$$
\left[\mathscr{D}, \mathscr{D}^{r}\right]=\operatorname{span}\left\{[X, Y]: X \in \mathscr{D}, Y \in \mathscr{D}^{r}\right\} .
$$

As in the classical case, we say that $\mathscr{D}$ is a bracket-generating distribution, if there exists an $r \geq 2$ such that $\mathscr{D}^{r}=\operatorname{Der} \mathscr{O}_{M}$. In this case $r$ is called the step of the distribution $\mathscr{D}$.

Suppose that $X, Y \in \mathscr{D}$ and consider the linear map on $\mathscr{D}$ as follows:

$$
F(X, Y)=-(-1)^{|X||Y|}[X, Y] \bmod \mathscr{D} .
$$

With respect to the above local basis $\left\{D_{i}, C_{a}, D_{\mu}, C_{\alpha}\right\}$ of $\operatorname{Der}_{M}$, if

$$
\begin{aligned}
& {\left[D_{i}, D_{j}\right]=D_{i j}^{k} D_{k}+D_{i j}^{d} C_{d}+\tilde{D}_{i j}^{v} D_{v}+\tilde{D}_{i j}^{\gamma} C_{\gamma},} \\
& {\left[D_{i}, D_{\xi}\right]=D_{i \xi}^{k} D_{k}+D_{i \xi}^{d} C_{d}+\tilde{D}_{i \xi}^{v} D_{v}+\tilde{D}_{i \xi}^{\gamma} C_{\gamma},} \\
& {\left[D_{\mu}, D_{j}\right]=D_{\mu j}^{k} D_{k}+D_{\mu j}^{d} C_{d}+\tilde{D}_{\mu j}^{v} D_{v}+\tilde{D}_{\mu j}^{\gamma} C_{\gamma},} \\
& {\left[D_{\mu}, D_{\xi}\right]=D_{\mu \xi}^{k} D_{k}+D_{\mu \xi}^{d} C_{d}+\tilde{D}_{\mu \xi}^{v} D_{v}+\tilde{D}_{\mu \xi}^{\gamma} C_{\gamma},}
\end{aligned}
$$

then, by using (2.3), we conclude that

$$
\begin{aligned}
& F\left(D_{j}, D_{i}\right)=D_{i j}^{d} C_{d}+\tilde{D}_{i j}^{\gamma} C_{\gamma} \bmod \mathscr{D}, \\
& F\left(D_{\xi}, D_{i}\right)=D_{i \xi}^{d} C_{d}+\tilde{D}_{i \xi}^{\gamma} C_{\gamma} \bmod \mathscr{D}, \\
& F\left(D_{j}, D_{\mu}\right)=D_{\mu j}^{d} C_{d}+\tilde{D}_{\mu j}^{\gamma} C_{\gamma} \bmod \mathscr{D}, \\
& F\left(D_{\xi}, D_{\mu}\right)=D_{\mu \xi}^{d} C_{d}+\tilde{D}_{\mu \xi}^{\gamma} C_{\gamma} \bmod \mathscr{D} .
\end{aligned}
$$

Each component $D_{b c}^{a}$ of $F$ is a superfunction on $U$.

Let $\bar{U}$ be an open subset of $M$ such that $U \cap \bar{U} \neq \emptyset$. If we change the basis of $\operatorname{Der} \mathscr{O}_{M}(U \cap \bar{U})$ to $\left\{\bar{D}_{i}, \bar{C}_{a}, \bar{D}_{\mu}, \bar{C}_{\alpha}\right\}$ then we have

$$
\begin{aligned}
& \bar{D}_{j}=f_{j}^{i} D_{i}+f_{j}^{\mu} D_{\mu}, \\
& \bar{D}_{v}=f_{v}^{i} D_{i}+f_{v}^{\mu} D_{\mu}, \\
& \bar{C}_{b}=f_{b}^{i} D_{i}+g_{b}^{a} C_{a}+f_{b}^{\mu} D_{\mu}+g_{b}^{\alpha} C_{\alpha}, \\
& \bar{C}_{\beta}=f_{\beta}^{i} D_{i}+g_{\beta}^{a} C_{a}+f_{\beta}^{\mu} D_{\mu}+g_{\beta}^{\alpha} C_{\alpha},
\end{aligned}
$$

where

$$
\left[\begin{array}{ll}
f_{j}^{i} & f_{j}^{\mu} \\
f_{v}^{i} & f_{v}^{\mu}
\end{array}\right] \text { and }\left[\begin{array}{ll}
g_{b}^{a} & g_{b}^{\alpha} \\
g_{\beta}^{a} & g_{\beta}^{\alpha}
\end{array}\right]
$$

are nonsingular supermatrices of smooth functions on $U \cap \bar{U}$. Both of these matrices are even. With respect to the basis $\left\{\bar{D}_{j}, \bar{C}_{b}, \bar{D}_{v}, \bar{C}_{\beta}\right\}$ on $\bar{U}$, if $\left\{\bar{D}_{k h}^{b}, \bar{D}_{k h}^{\beta}, \ldots, \bar{D}_{\xi_{\rho}}^{\beta}\right\}$ are the local components of $F$, then we have

$$
\left[\begin{array}{ll}
\bar{D}_{k h}^{b} & \bar{D}_{k h}^{\beta} \\
\bar{D}_{\xi h}^{b} & \bar{D}_{\xi h}^{\beta} \\
\bar{D}_{k \rho}^{b} & \bar{D}_{k \rho}^{\beta} \\
\bar{D}_{\xi \rho}^{b} & \bar{D}_{\xi \rho}^{\beta}
\end{array}\right]\left[\begin{array}{cc}
g_{b}^{a} & g_{b}^{\alpha} \\
g_{\beta}^{a} & g_{\beta}^{\alpha}
\end{array}\right]=\left[\begin{array}{cccc}
f_{h}^{j} & 0 & f_{h}^{\mu} & 0 \\
0 & f_{\rho}^{\mu} & 0 & f_{\rho}^{j} \\
f_{\rho}^{j} & 0 & f_{\rho}^{\mu} & 0 \\
0 & f_{h}^{\mu} & 0 & f_{h}^{j}
\end{array}\right]\left[\begin{array}{cccc}
f_{k}^{i} & 0 & f_{k}^{v} & 0 \\
0 & f_{\xi}^{v} & 0 & -f_{\xi}^{i} \\
0 & -f_{k}^{v} & 0 & f_{k}^{i} \\
f_{\xi}^{i} & 0 & f_{\xi}^{v} & 0
\end{array}\right]\left[\begin{array}{cc}
D_{i j}^{a} & D_{i j}^{\alpha} \\
D_{v \mu}^{a} & D_{v \mu}^{\alpha} \\
D_{v j}^{a} & D_{v j}^{\alpha} \\
D_{i \mu}^{a} & D_{i \mu}^{\alpha}
\end{array}\right]
$$


Since $\left[\begin{array}{cc}f_{j}^{i} & f_{j}^{\mu} \\ f_{v}^{i} & f_{v}^{\mu}\end{array}\right]$ is invertible at $x \in U \cap \bar{U}$, we see that $\left[\begin{array}{cc}f_{j}^{i} & 0 \\ 0 & f_{v}^{\mu}\end{array}\right]$ is invertible and from (2.5) we conclude that if

$$
D(x)=\left[\begin{array}{ccccccccc}
D_{12}^{p+q+1} & D_{13}^{p+q+1} & \ldots & D_{1 p+q}^{p+q+1} & D_{23}^{p+q+1} & \ldots & D_{2 p+q}^{p+q+1} & \ldots & D_{p+q-1 p+q}^{p+q+1} \\
\vdots & \vdots & & \vdots & \vdots & & \vdots & & \vdots \\
D_{12}^{m+n} & D_{13}^{m+n} & \ldots & D_{1 p+q}^{m+n} & D_{23}^{m+n} & \ldots & D_{2 p+q}^{m+n} & \ldots & D_{p+q-1 p+q}^{m+n}
\end{array}\right](x)
$$

then $\operatorname{rank} D(x)=\operatorname{rank} \bar{D}(x)$.

Now we can define the rank of $F$, which is related to its coefficients matrix. Before doing this, in view of (2.4), we note that the submatrices

$$
\left[\begin{array}{ll}
D_{i j}^{a}(x) & D_{\mu \nu}^{a}(x) \\
\tilde{D}_{i j}^{\alpha}(x) & \tilde{D}_{\mu \nu}^{\alpha}(x)
\end{array}\right] \text { and }\left[\begin{array}{cc}
D_{i \mu}^{a}(x) & D_{\mu i}^{a}(x) \\
\tilde{D}_{i \mu}^{\alpha}(x) & \tilde{D}_{\mu i}^{\alpha}(x)
\end{array}\right]
$$

are even and odd respectively. The rank of the first submatrix can be defined but for the second submatrix, since $D_{\mu i}^{a}(x)$ and $\tilde{D}_{i \mu}^{\alpha}(x)$ are even, we consider the matrix $\left[\begin{array}{cc}D_{\mu i}^{a}(x) & D_{i \mu}^{a}(x) \\ \tilde{D}_{\mu i}^{\alpha}(x) & \tilde{D}_{i \mu}^{\alpha}(x)\end{array}\right]$ to define its rank. Now set

$$
r:=\operatorname{rank}\left[\begin{array}{cc}
D_{i j}^{a}(x) & D_{\mu v}^{a}(x) \\
\tilde{D}_{i j}^{\alpha}(x) & \tilde{D}_{\mu v}^{\alpha}(x)
\end{array}\right] \text { and } s:=\operatorname{rank}\left[\begin{array}{cc}
D_{\mu i}^{a}(x) & D_{i \mu}^{a}(x) \\
\tilde{D}_{\mu i}^{\alpha}(x) & \tilde{D}_{i \mu}^{\alpha}(x)
\end{array}\right]
$$

where $i, j=1, \ldots, p, a=p+1, . ., m$ and $\mu, v=1, \ldots, q, \alpha=q+1, \ldots, n$. Thus we define

$$
\operatorname{rankF}(x)=(r, s) \text {. }
$$

If $\left(q_{\bar{a}}, \xi_{\bar{\mu}}\right)$ are local supercoordinates on a coordinate neighborhood $U$ of $x \in M,(\bar{a}=1, \ldots, m, \bar{\mu}=1, \ldots, n)$, then $\mathscr{D}$ is locally given by the graded 1 -forms

$$
\begin{aligned}
& \phi_{\bar{b}}=\phi_{\bar{b}}^{\bar{a}} d q_{\bar{a}}+\tilde{\phi}_{\bar{b}}^{\bar{\mu}} d \xi_{\bar{\mu}}=0, \quad \bar{b}=1, \ldots, p \\
& \phi_{\bar{\alpha}}=\phi_{\bar{\alpha}}^{\bar{a}} d q_{\bar{a}}+\tilde{\phi}_{\bar{\alpha}}^{\bar{\mu}} d \xi_{\bar{\mu}}=0, \quad \bar{\alpha}=1, \ldots, q .
\end{aligned}
$$

Since $\mathscr{D}$ is a distribution of rank $(p, q)$, we may assume that the submatrices $\left(\phi_{\bar{b}}^{\bar{a}}\right), 1 \leq \bar{a}, \bar{b} \leq p$, and $\left(\tilde{\phi}_{\bar{\alpha}}^{\bar{\mu}}\right), 1 \leq \bar{\alpha}, \bar{\mu} \leq q$ are invertible. Let the matrix $\psi=\left(\psi_{\bullet}\right)$ denotes the inverse of the matrix $\left(\begin{array}{cc}\phi_{\bar{b}}^{\bar{a}} & \tilde{\phi}_{\bar{b}}^{\bar{\mu}} \\ \phi_{\bar{\alpha}}^{\bar{a}} & \tilde{\phi}_{\bar{\alpha}}^{\bar{\mu}}\end{array}\right), 1 \leq \bar{a}, \bar{b} \leq p, 1 \leq \bar{\alpha}, \bar{\mu} \leq q$ and suppose

$$
\bar{\phi}_{\bar{a}}=\psi_{\bar{a}}^{\bar{b}} \phi_{\bar{b}}+\tilde{\phi}_{\bar{a}}^{\bar{\mu}} \phi_{\bar{\mu}}, \quad \bar{\phi}_{\bar{\alpha}}=\phi_{\bar{\alpha}}^{\bar{b}} \phi_{\bar{b}}+\tilde{\phi}_{\bar{\alpha}}^{\bar{\mu}} \phi_{\bar{\mu}}
$$

Therefore, the new notation

$$
\begin{aligned}
& y_{a}=q_{a}, x_{i}=q_{i}, i=1, \ldots, p, \quad a=p+1, \ldots, m, \\
& \zeta_{\alpha}=\xi_{\alpha}, \eta_{\mu}=\xi_{\mu}, \mu=1, \ldots, q, \quad \alpha=q+1, \ldots, n,
\end{aligned}
$$

for the coordinates, may be performed to bring the local basis of $\Omega^{1}(\mathscr{M})$ into the form $\left\{d x_{i}, d \eta_{\mu}, d y_{a}+r_{i}^{a} d x_{i}+r_{\mu}^{a} d \eta_{\mu}, d \zeta_{\alpha}+r_{i}^{\alpha} d x_{i}+\right.$ $\left.r_{\mu}^{\alpha} d \eta_{\mu}\right\}$. It is easy to check that

$$
\begin{aligned}
\frac{\delta}{\delta x_{i}} & :=\frac{\partial}{\partial x_{i}}-r_{i}^{a} \frac{\partial}{\partial y_{a}}-r_{i}^{\alpha} \frac{\partial}{\partial \zeta_{\alpha}}, i=1, \ldots, p, \\
\frac{\delta}{\delta \eta_{\mu}} & :=\frac{\partial}{\partial \eta_{\mu}}+r_{\mu}^{a} \frac{\partial}{\partial y_{a}}-r_{\mu}^{\alpha} \frac{\partial}{\partial \zeta_{\alpha}}, \mu=1, \ldots, q,
\end{aligned}
$$

are (respectively even and odd) generators of $\mathscr{D}$ on $U$ and $\left\{\delta / \delta x_{i}, \delta / \delta \eta_{\mu}, \partial / \partial y_{a}, \partial / \partial \zeta_{\alpha}\right\}$ is a local basis for $\operatorname{Der}\left(\mathscr{O}_{M}(U)\right)$, (see also [4, 5]). With respect to this basis, if we put

$$
\begin{aligned}
& F\left(\frac{\delta}{\delta x_{j}}, \frac{\delta}{\delta x_{i}}\right)=F_{i}{ }_{j}^{a} \frac{\partial}{\partial y_{a}}+\tilde{F}_{i}{ }_{j}^{\alpha} \frac{\partial}{\partial \zeta_{\alpha}} \bmod \mathscr{D}, \\
& F\left(\frac{\delta}{\delta x_{j}}, \frac{\delta}{\delta \eta_{v}}\right)=F_{v j}^{a} \frac{\partial}{\partial y_{a}}+\tilde{F}_{v}^{\alpha} \frac{\partial}{\partial \zeta_{\alpha}} \bmod \mathscr{D}, \\
& F\left(\frac{\delta}{\delta \eta_{\mu}}, \frac{\delta}{\delta x_{i}}\right)=F_{i}{ }^{a} \frac{\partial}{\partial y_{a}}+\tilde{F}_{i \mu}^{\alpha} \frac{\partial}{\partial \zeta_{\alpha}} \bmod \mathscr{D}, \\
& F\left(\frac{\delta}{\delta \eta_{\mu}}, \frac{\delta}{\delta \eta_{v}}\right)=F_{v}{ }^{a} \frac{\partial}{\partial y_{a}}+\tilde{F}_{v \mu}^{\alpha} \frac{\partial}{\partial \zeta_{\alpha}} \bmod \mathscr{D},
\end{aligned}
$$


then by using (2.3) and (2.6), we deduce that

$$
\begin{aligned}
& F_{i}{ }^{a} \frac{\partial}{\partial y_{a}}+\tilde{F}_{i}{ }_{j}^{\alpha} \frac{\partial}{\partial \zeta_{\alpha}}=\left[\frac{\delta}{\delta x_{i}}, \frac{\delta}{\delta x_{j}}\right]=\left(\frac{\delta r_{i}^{a}}{\delta x_{j}}-\frac{\delta r_{j}^{a}}{\delta x_{i}}\right) \frac{\partial}{\partial y_{a}}+\left(\frac{\delta r_{i}^{\alpha}}{\delta x_{j}}-\frac{\delta r_{j}^{\alpha}}{\delta x_{i}}\right) \frac{\partial}{\partial \zeta_{\alpha}} \bmod \mathscr{D}, \\
& F_{v j}^{a} \frac{\partial}{\partial y_{a}}+\tilde{F}_{v}^{\alpha} \frac{\partial}{\partial \zeta_{\alpha}}=\left[\frac{\delta}{\delta \eta_{v}}, \frac{\delta}{\delta x_{j}}\right]=\left(-\frac{\delta r_{v}^{a}}{\delta x_{j}}-\frac{\delta r_{j}^{a}}{\delta \eta_{v}}\right) \frac{\partial}{\partial y_{a}}+\left(\frac{\delta r_{v}^{\alpha}}{\delta x_{j}}-\frac{\delta r_{j}^{\alpha}}{\delta \eta_{v}}\right) \frac{\partial}{\partial \zeta_{\alpha}} \bmod \mathscr{D}, \\
& F_{i}{ }^{a} \frac{\partial}{\partial y_{a}}+\tilde{F}_{i}^{\alpha}{ }^{\alpha} \frac{\partial}{\partial \zeta_{\alpha}}=\left[\frac{\delta}{\delta x_{i}}, \frac{\delta}{\delta \eta_{\mu}}\right]=\left(\frac{\delta r_{i}^{a}}{\delta \eta_{\mu}}+\frac{\delta r_{\mu}^{a}}{\delta x_{i}}\right) \frac{\partial}{\partial y_{a}}+\left(\frac{\delta r_{i}^{\alpha}}{\delta \eta_{\mu}}-\frac{\delta r_{\mu}^{\alpha}}{\delta x_{i}}\right) \frac{\partial}{\partial \zeta_{\alpha}} \bmod \mathscr{D}, \\
& F_{v \mu}^{a} \frac{\partial}{\partial y_{a}}+\tilde{F}_{v}^{\alpha} \frac{\partial}{\partial \zeta_{\alpha}}=\left[\frac{\delta}{\delta \eta_{v}}, \frac{\delta}{\delta \eta_{\mu}}\right]=\left(\frac{\delta r_{v}^{a}}{\delta \eta_{\mu}}+\frac{\delta r_{\mu}^{a}}{\delta \eta_{v}}\right) \frac{\partial}{\partial y_{a}}+\left(-\frac{\delta r_{v}^{\alpha}}{\delta \eta_{\mu}}-\frac{\delta r_{\mu}^{\alpha}}{\delta \eta_{v}}\right) \frac{\partial}{\partial \zeta_{\alpha}} \bmod \mathscr{D} .
\end{aligned}
$$

Now let us consider a distribution $\mathscr{D}$ of corank one on $\mathscr{M}$. For each $z \in M$, there are two cases.

Case1. Let $\operatorname{rank} \mathscr{D}(z)=(m-1, n)$. Then there exist a coordinate system $\left(x_{i}, t, \eta_{\mu}\right), i=1, \ldots, m-1, \mu=1, \ldots, n$, defined in a neighborhood $U$ of $z$, such that $\mathscr{D}$ is locally given by

$$
d t+r_{i} d x_{i}+r_{\mu} d \eta_{\mu}=0
$$

Case2. Let $\operatorname{rank} \mathscr{D}(z)=(m, n-1)$. Then there exist a coordinate system $\left(x_{j}, \eta_{v}, \theta\right), j=1, \ldots, m, v=1, \ldots, n-1$ defined in a neighborhood $U$ of $z$, such that $\mathscr{D}$ is locally given by

$$
d \theta+r_{j} d x_{j}+r_{v} d \eta_{v}=0 .
$$

Note that in the first case, (2.8) becomes

$$
\begin{aligned}
& F_{i j} \frac{\partial}{\partial t}=\left[\frac{\delta}{\delta x_{i}}, \frac{\delta}{\delta x_{j}}\right]=\left(\frac{\delta r_{i}}{\delta x_{j}}-\frac{\delta r_{j}}{\delta x_{i}}\right) \frac{\partial}{\partial t} \bmod \mathscr{D}, \\
& F_{v j} \frac{\partial}{\partial t}=\left[\frac{\delta}{\delta \eta_{v}}, \frac{\delta}{\delta x_{j}}\right]=\left(-\frac{\delta r_{j}}{\delta \eta_{v}}-(-1)^{|t|} \frac{\delta r_{v}}{\delta x_{j}}\right) \frac{\partial}{\partial t} \bmod \mathscr{D}, \\
& F_{i \mu} \frac{\partial}{\partial t}=\left[\frac{\delta}{\delta x_{i}}, \frac{\delta}{\delta \eta_{\mu}}\right]=\left(\frac{\delta r_{i}}{\delta \eta_{\mu}}+(-1)^{|t|} \frac{\delta r_{\mu}}{\delta x_{i}}\right) \frac{\partial}{\partial t} \bmod \mathscr{D}, \\
& F_{v \mu} \frac{\partial}{\partial t}=\left[\frac{\delta}{\delta \eta_{v}}, \frac{\delta}{\delta \eta_{\mu}}\right]=\left((-1)^{|t|} \frac{\delta r_{v}}{\delta \eta_{\mu}}+(-1)^{|t|} \frac{\delta r_{\mu}}{\delta \eta_{v}}\right) \frac{\partial}{\partial t} \bmod \mathscr{D},
\end{aligned}
$$

where $F_{i j}, F_{v j}, F_{i \mu}$ and $F_{v \mu}$ are the local components of $F$ with respect to the local basis $\left\{\delta / \delta x_{i}, \delta / \delta x_{\mu}, \partial / \partial t\right\}$.

\section{Bracket-generating distribution of step 2}

In this section, we want to find the conditions under which a distribution $\mathscr{D}$ is bracket-generating of step 2. As mentioned in the previous section, we attach to $\mathscr{D}$ a linear map $F$ on $\mathscr{D}$ defined by the Lie bracket of graded vector fields of the sections of $\mathscr{D}$. We will have several types of possibilities for the rank of $F$. Using this, we find conditions to describe the problem.

Theorem 3.1. Let $\mathscr{D}$ be a distribution of rank $(p, q)(p<m, q<n)$ on an $(m, n)$-dimensional graded manifold $\mathscr{M}$ such that

$$
m-p \leq \frac{p(p-1)}{2}+\frac{q(q-1)}{2}, n-q \leq \frac{q(q-1)}{2},
$$

Then $\mathscr{D}$ is a bracket-generating distribution of step 2, if and only if, the linear map $F$ associated to $\mathscr{D}$ is of constant rank $(m-p, n-q)$ on $\mathscr{M}$.

Proof. Let $x \in M$. Suppose $\mathscr{D}$ is a bracket-generating distribution of step 2 and let $\left\{\delta / \delta x_{i}, \delta / \delta \eta_{\mu}, \partial / \partial y_{a}, \partial / \partial \zeta_{\alpha}\right\}$ be a basis of $D e r \mathscr{O}_{M}(U)$ in a coordinate neighborhood $U$ of $x$. Then $\operatorname{rank}[\mathscr{D}, \mathscr{D}](x)=(m-p, n-q)$. This means that the number of linearly independent graded vector fields of the set $\left\{\left[\frac{\delta}{\delta x_{i}}, \frac{\delta}{\delta x_{j}}\right],\left[\frac{\delta}{\delta \eta_{\mu}}, \frac{\delta}{\delta \eta_{v}}\right], 1 \leq i, j \leq p, 1 \leq \mu, v \leq q\right\}$, (respectively $\left.\left\{\left[\frac{\delta}{\delta x_{i}}, \frac{\delta}{\delta \eta_{\mu}}\right], 1 \leq i \leq p, 1 \leq \mu \leq q\right\}\right)$ is $m-p$ (respectively $n-q$ ). Therefore the coefficient matrix, the matrix consisting of the coefficients of the Lie brackets of graded vector fields $\left\{\left[\frac{\delta}{\delta x_{i}}, \frac{\delta}{\delta x_{j}}\right],\left[\frac{\delta}{\delta \eta_{\mu}}, \frac{\delta}{\delta \eta_{v}}\right]\right\}$ at the point $x$, denoted by

$$
\left[\begin{array}{ll}
D_{i j}^{a}(x) & D_{\mu v}^{a}(x) \\
\tilde{D}_{i j}^{\alpha}(x) & \tilde{D}_{\mu v}^{\alpha}(x)
\end{array}\right], \begin{aligned}
& a=1, \ldots, m-p \\
& \alpha=1, \ldots, n-q
\end{aligned},(\bmod \mathscr{D}),
$$

having the rank $m-p$, is invertible. Similarly, the coefficient matrix

$$
\left[\begin{array}{l}
D_{i \mu}^{a}(x) \\
\tilde{D}_{i \mu}^{\alpha}(x)
\end{array}\right], \begin{gathered}
a=1, \ldots, m-p \\
\alpha=1, \ldots, n-q
\end{gathered},(\quad \bmod \mathscr{D}),
$$

the matrix consisting of the coefficients of the Lie brackets of graded vector fields $\left\{\left[\frac{\delta}{\delta x_{i}}, \frac{\delta}{\delta \eta_{\mu}}\right]\right\}$ at the point $x$, has rank $n-q$, (i.e. $n-q=$ $\operatorname{rank}\left(\begin{array}{l}\tilde{D}_{i \mu}^{\alpha}(x) \\ D_{i \mu}^{a}(x)\end{array}\right)$, and this matrix is even). Hence associated with $F$ is the graded vector field, represented by the matrix $\left(\begin{array}{c}D_{b c}^{a}(x) \\ \tilde{D}_{e f}^{\alpha}(x)\end{array}\right),(\bmod \mathscr{D})$, relative to the above basis. It is clear that $\operatorname{rank} F(x)=(m-p, n-q)$. 
Conversely, suppose that $x \in M$ and $\operatorname{rankF}(x)=(m-p, n-q)$ on $\mathscr{M}$. Let $\left\{\delta / \delta x_{i}, \delta / \delta \eta_{\mu}, \partial / \partial y_{a}, \partial / \partial \zeta_{\alpha}\right\}$ be a basis of $D e r \mathscr{O}_{M}(U)$ in a coordinate neighborhood $U$ of $x$. Consider the coefficient matrix of the graded vector fields $F\left(\frac{\delta}{\delta x_{i}}, \frac{\delta}{\delta x_{j}}\right)$ and $F\left(\frac{\delta}{\delta \eta_{\mu}}, \frac{\delta}{\delta \eta_{\nu}}\right)$, which is even and denoted by

$$
\left[\begin{array}{ll}
F_{i j}^{a}(x) & F_{\mu \nu}^{a}(x) \\
\tilde{F}_{i j}^{\alpha}(x) & \tilde{F}_{\mu v}^{\alpha}(x)
\end{array}\right]
$$

Note that its rank is $m-p$, otherwise $F$ would not be a map of the given rank. Thus there are two non-negative integers $r$ and $s$ such that $r+s=m-p$ and $\operatorname{rank}\left(F_{i j}^{a}(x)\right)=r, \operatorname{rank}\left(\tilde{F}_{\mu v}^{\alpha}(x)\right)=s$. Hence we may assume that the submatrices $G=\left(F_{i^{\prime} j^{\prime}}^{a^{\prime}}(x)\right), 1 \leq a^{\prime}, j^{\prime}-1 \leq r, i^{\prime}<j^{\prime}$ and $J=\left(\tilde{F}_{\mu^{\prime} v^{\prime}}^{\alpha^{\prime}}(x)\right), 1 \leq \alpha^{\prime}, v^{\prime}-1 \leq s, \mu^{\prime}<v^{\prime}$, are both invertible. Therefore, the submatrix,

$$
\left[\begin{array}{cc}
G & H \\
I & J
\end{array}\right]=\left[\begin{array}{cc}
F_{i^{\prime} j^{\prime}}^{a^{\prime}}(x) & F_{\mu^{\prime} y^{\prime}}^{a^{\prime}}(x) \\
\tilde{F}_{i^{\prime} j^{\prime}}^{\alpha^{\prime}}(x) & \tilde{F}_{\mu^{\prime} v^{\prime}}^{\alpha^{\prime}}(x)
\end{array}\right], \begin{array}{r}
1 \leq a^{\prime}, j^{\prime}-1 \leq r \\
i^{\prime}<j^{\prime}
\end{array} \underset{\mu^{\prime}<v^{\prime}}{1 \leq \alpha^{\prime}, v^{\prime}-1 \leq s}
$$

is invertible.

Similarly, consider the coefficient matrix of the graded vector fields $F\left(\frac{\delta}{\delta x_{i}}, \frac{\delta}{\delta \eta_{\mu}}\right)$, which is odd and its rank is $n-q$. We denote it by

$$
\left[\begin{array}{c}
F_{i \mu}^{a}(x) \\
\tilde{F}_{i \mu}^{\alpha}(x)
\end{array}\right], 1 \leq a \leq m-p, 1 \leq \alpha \leq n-q .
$$

Since $\operatorname{rank}\left(\tilde{F}_{i \mu}^{\alpha}(x)\right)=n-q$, we may assume that the submatrice $\left(\tilde{F}_{i^{\prime} \mu^{\prime}}^{\alpha}(x)\right), 1 \leq \mu^{\prime}-1 \leq n-q, i^{\prime}<\mu^{\prime}$, is invertible. We thus consider

$$
\left[\begin{array}{c}
F_{i^{\prime} \mu^{\prime}}^{a}(x) \\
\tilde{F}_{i^{\prime} \mu^{\prime}}^{\alpha}(x)
\end{array}\right], 1 \leq \mu^{\prime}-1 \leq n-q, i^{\prime}<\mu^{\prime} .
$$

Given the matrices (3.3) and (3.4), we may change the generators of $\operatorname{Der} \mathscr{O} \mathscr{M}$ to $\left\{\delta / \delta x_{i}, \delta / \delta \eta_{\mu}, Y_{b}, Z_{v}\right\}, b=1, \ldots, m-p ; v=1, \ldots, n-q$,

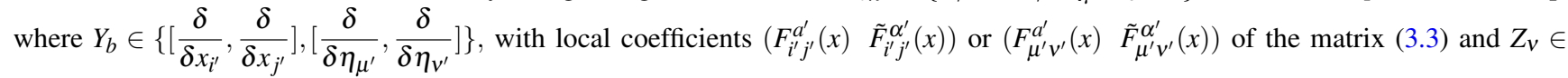
$\left\{\left[\frac{\delta}{\delta x_{i^{\prime}}}, \frac{\delta}{\delta \eta_{\mu^{\prime}}}\right]\right\}$, with local coefficients $\left(F_{i^{\prime} \mu^{\prime}}^{a^{\prime}}(x) \quad \tilde{F}_{i^{\prime} \mu^{\prime}}^{\alpha^{\prime}}(x)\right)$ of the matrix (3.3). Thus $\mathscr{D}$ is bracket-generating of step 2.

By using Theorem (3.1) we can easily prove the following theorems.

Theorem 3.2. Let $\mathscr{M}$ be an $(m, n)$ dimensional graded manifold. Suppose that $\mathscr{D}$ is a distribution of rank $(m-1, n)$. Then $\mathscr{D}$ is bracketgenerating of step 2 , if and only if, for the linear map $F=F_{0}+F_{1}$ associated to $\mathscr{D}, F_{0} \neq 0$ on $\mathscr{M}$.

Proof. Since $\operatorname{rank} \mathscr{D}(z)=(m-1, n)$, there exist a coordinate system $\left(x_{i}, t, \eta_{\mu}\right), i=1, \ldots, m-1, \mu=1, \ldots, n$, defined in a neighborhood $U$ of $z$, such that $\mathscr{D}$ is locally given by $\left\{\delta / \delta x_{i}, \delta / \delta \eta_{\mu}\right\}$ and $\left\{\delta / \delta x_{i}, \delta / \delta \eta_{\mu}, \partial / \partial t\right\}$ is a local basis for $\operatorname{Der} \mathscr{O}_{M}$. Therefore, according to the Theorem 3.1, the coefficient matrix,

$$
\left[D_{i j}^{1}(x) \quad D_{\mu \nu}^{1}(x)\right], \quad(\bmod \mathscr{D})
$$

has the rank 1 . Hence $F_{0} \neq 0$.

Theorem 3.3. Let $\mathscr{M}$ be an $(m, n)$-dimensional graded manifold. Suppose that $\mathscr{D}$ is a distribution of rank $(m, n-1)$. Then $\mathscr{D}$ is bracket-generating of step 2 , if and only if, for the linear map $F=F_{0}+F_{1}$ associated to $\mathscr{D}, F_{1} \neq 0$ on $\mathscr{M}$.

Proof. Since $\operatorname{rank} \mathscr{D}(z)=(m, n-1)$, there exist a coordinate system $\left(x_{i}, \eta_{\mu}, \theta\right), i=1, \ldots, m, \mu=1, \ldots, n-1$, defined in a neighborhood $U$ of $z$, such that $\mathscr{D}$ is locally given by $\left\{\delta / \delta x_{i}, \delta / \delta \eta_{\mu}\right\}$ and $\left\{\delta / \delta x_{i}, \delta / \delta \eta_{\mu}, \partial / \partial \theta\right\}$ is a local basis for $\operatorname{Der} \mathscr{O}_{M}$. Therefore, according to the Theorem 3.1, the coefficient matrix,

$$
\left[\tilde{D}_{i \mu}^{1}(x)\right], \quad(\bmod \mathscr{D})
$$

has the rank $n$. Hence $F_{1} \neq 0$.

Theorem 3.4. Let $\mathscr{M}$ be an $(m, n)$ dimensional graded manifold. Suppose that $\mathscr{D}$ is a distribution of rank $(0, n)$. Then $\mathscr{D}$ is bracket-generating of step 2 , if and only if, for the linear map $F=F_{0}+F_{1}$ associated to $\mathscr{D}$, rank $F_{0}=m$ on $\mathscr{M}$.

Proof. The details are the same as those given in the proof of Theorem 3.1.

Example 3.5. Consider the graded manifold $\mathscr{M}=R^{3 \mid 1}$. Let $\left(x_{i}, t, \eta\right), i=1, \ldots, 2$ be local supercoordinates on a coordinate neighborhood $U$ of $x \in R^{3}$. Suppose that $\mathscr{D}$ is the distribution spanned by $\frac{\delta}{\delta x_{1}}, \frac{\delta}{\delta x_{2}}$ and $\frac{\delta}{\delta \eta}$ where

$$
\frac{\delta}{\delta \eta}=\frac{\partial}{\partial \eta}+\eta \frac{\partial}{\partial t}, \quad \frac{\delta}{\delta x_{1}}=\frac{\partial}{\partial x_{1}}-\frac{x_{2}}{2} \frac{\partial}{\partial t}, \quad \frac{\delta}{\delta x_{2}}=\frac{\partial}{\partial x_{2}}+\frac{x_{1}}{2} \frac{\partial}{\partial t} .
$$

A simple calculation shows that $\left[\frac{\delta}{\delta x_{1}}, \frac{\delta}{\delta x_{2}}\right]=\frac{\partial}{\partial t}$ and $\left\{\frac{\delta}{\delta x_{1}}, \frac{\delta}{\delta x_{2}}, \frac{\partial}{\partial t}, \frac{\delta}{\delta \eta}\right\}$ is a basis of Der $\mathscr{O}_{R^{3}}(U)$. Thus $\mathscr{D}$ is bracket-generating of step 2. 
Example 3.6. Consider the graded manifold $\mathscr{M}=R^{4 \mid 4}$. Let $\left(x_{i}, \eta_{\mu}\right), i, \mu=1, \ldots, 4$ be local supercoordinates on a coordinate neighborhood $U$ of $x \in R^{4}$. Suppose that $\mathscr{D}$ is the distribution (see [7]) spanned by $\frac{\delta}{\delta \eta_{1}}, \frac{\delta}{\delta \eta_{2}}, \frac{\delta}{\delta \eta_{3}}$ and $\frac{\delta}{\delta \eta_{4}}$, where

$$
\begin{aligned}
& \frac{\delta}{\delta \eta_{1}}=\frac{\partial}{\partial \eta_{1}}-i \eta_{3} \frac{\partial}{\partial x_{1}}-i \eta_{4} \frac{\partial}{\partial x_{2}}-\eta_{4} \frac{\partial}{\partial x_{3}}-i \eta_{3} \frac{\partial}{\partial x_{4}} \\
& \frac{\delta}{\delta \eta_{2}}=\frac{\partial}{\partial \eta_{2}}-i \eta_{4} \frac{\partial}{\partial x_{1}}-i \eta_{3} \frac{\partial}{\partial x_{2}}+\eta_{3} \frac{\partial}{\partial x_{3}}+i \eta_{4} \frac{\partial}{\partial x_{4}} \\
& \frac{\delta}{\delta \eta_{3}}=\frac{\partial}{\partial \eta_{3}}-i \eta_{1} \frac{\partial}{\partial x_{1}}-i \eta_{2} \frac{\partial}{\partial x_{2}}+\eta_{2} \frac{\partial}{\partial x_{3}}-i \eta_{1} \frac{\partial}{\partial x_{4}} \\
& \frac{\delta}{\delta \eta_{4}}=\frac{\partial}{\partial \eta_{4}}-i \eta_{2} \frac{\partial}{\partial x_{1}}-i \eta_{1} \frac{\partial}{\partial x_{2}}-\eta_{1} \frac{\partial}{\partial x_{3}}+i \eta_{2} \frac{\partial}{\partial x_{4}}
\end{aligned}
$$

Here $i=\sqrt{-1}$. Thus the vector fields $\left[\frac{\delta}{\delta \eta_{1}}, \frac{\delta}{\delta \eta_{1}}\right],\left[\frac{\delta}{\delta \eta_{1}}, \frac{\delta}{\delta \eta_{2}}\right],\left[\frac{\delta}{\delta \eta_{3}}, \frac{\delta}{\delta \eta_{3}}\right]$, and $\left[\frac{\delta}{\delta \eta_{3}}, \frac{\delta}{\delta \eta_{4}}\right]$ are zero and

$$
\begin{aligned}
& {\left[\frac{\delta}{\delta \eta_{1}}, \frac{\delta}{\delta \eta_{3}}\right]=-2 i \frac{\partial}{\partial x_{1}}-2 i \frac{\partial}{\partial x_{4}}, \quad\left[\frac{\delta}{\delta \eta_{1}}, \frac{\delta}{\delta \eta_{4}}\right]=-2 i \frac{\partial}{\partial x_{2}}-2 \frac{\partial}{\partial x_{3}}} \\
& {\left[\frac{\delta}{\delta \eta_{2}}, \frac{\delta}{\delta \eta_{3}}\right]=-2 i \frac{\partial}{\partial x_{2}}+2 \frac{\partial}{\partial x_{3}}, \quad\left[\frac{\delta}{\delta \eta_{2}}, \frac{\delta}{\delta \eta_{4}}\right]=-2 i \frac{\partial}{\partial x_{1}}+2 i \frac{\partial}{\partial x_{4}} .}
\end{aligned}
$$

In the notation used in Theorem 3.1, all of the entries $D_{i j}^{a}, \tilde{D}_{i j}^{\alpha}, \tilde{D}_{\mu \nu}^{\alpha}, D_{i \mu}^{a}, \tilde{D}_{i \mu}^{\alpha}$ of the coefficient matrix except $D_{\mu \nu}^{a}$ are zero and

$$
\left[D_{\mu \nu}^{a}\right]=\left[\begin{array}{cccccc}
0 & -2 i & 0 & 0 & -2 i & 0 \\
0 & 0 & -2 i & -2 i & 0 & 0 \\
0 & 0 & -2 & 2 & 0 & 0 \\
0 & -2 i & 0 & 0 & +2 i & 0
\end{array}\right]
$$

So we have rank $\left(D_{\mu v}^{a}\right)=4$, and we conclude from Corollary 3.4, that $\mathscr{D}$ is a bracket-generating distribution of step 2. By calculation we have

$$
\begin{aligned}
& \frac{1}{4} i\left(\left(\frac{\delta}{\delta \eta_{1}}+\left[\frac{\delta}{\delta \eta_{1}}, \frac{\delta}{\delta \eta_{3}}\right]\right)+\left(\frac{\delta}{\delta \eta_{1}}+\left[\frac{\delta}{\delta \eta_{2}}, \frac{\delta}{\delta \eta_{4}}\right]\right)-2\left(\frac{\delta}{\delta \eta_{1}}+\left[\frac{\delta}{\delta \eta_{1}}, \frac{\delta}{\delta \eta_{1}}\right]\right)\right)=\frac{\partial}{\partial x_{1}} \\
& \frac{1}{4} i\left(\left(\frac{\delta}{\delta \eta_{1}}+\left[\frac{\delta}{\delta \eta_{2}}, \frac{\delta}{\delta \eta_{3}}\right]\right)+\left(\frac{\delta}{\delta \eta_{1}}+\left[\frac{\delta}{\delta \eta_{1}}, \frac{\delta}{\delta \eta_{4}}\right]\right)-2\left(\frac{\delta}{\delta \eta_{1}}+\left[\frac{\delta}{\delta \eta_{1}}, \frac{\delta}{\delta \eta_{1}}\right]\right)\right)=\frac{\partial}{\partial x_{2}} \\
& \frac{1}{4}\left(\left(\frac{\delta}{\delta \eta_{1}}+\left[\frac{\delta}{\delta \eta_{2}}, \frac{\delta}{\delta \eta_{3}}\right]\right)-\left(\frac{\delta}{\delta \eta_{1}}+\left[\frac{\delta}{\delta \eta_{1}}, \frac{\delta}{\delta \eta_{4}}\right]\right)\right)=\frac{\partial}{\partial x_{3}} \\
& \frac{1}{4} i\left(\left(\frac{\delta}{\delta \eta_{1}}+\left[\frac{\delta}{\delta \eta_{1}}, \frac{\delta}{\delta \eta_{3}}\right]\right)-\left(\frac{\delta}{\delta \eta_{1}}+\left[\frac{\delta}{\delta \eta_{2}}, \frac{\delta}{\delta \eta_{4}}\right]\right)\right)=\frac{\partial}{\partial x_{4}}
\end{aligned}
$$

Example 3.7. Let $\mathscr{M}=R^{3 \mid 1}$ equiped with local supercoordinates $\left(x_{1}, x_{2}, x_{3}, \eta\right)$ and $\mathscr{D}$ be the distribution spanned by $\left\{\frac{\delta}{\delta x_{1}}=\frac{\partial}{\partial x_{1}}\right.$, $\frac{\delta}{\delta x_{2}}=$ $\left.\frac{\partial}{\partial x_{2}}+\left(x_{1}\right)^{2} \frac{\partial}{\partial x_{3}}, \frac{\delta}{\delta \eta}=\frac{\partial}{\partial \eta}\right\}$. In this case we have

$$
\begin{array}{r}
{\left[\frac{\delta}{\delta x_{1}}, \frac{\delta}{\delta \eta}\right]=\left[\frac{\delta}{\delta x_{2}}, \frac{\delta}{\delta \eta}\right]=0} \\
{\left[\frac{\delta}{\delta x_{1}}, \frac{\delta}{\delta x_{2}}\right]=2 x_{1} \frac{\partial}{\partial x_{3}}} \\
{\left[\frac{\delta}{\delta x_{1}},\left[\frac{\delta}{\delta x_{1}}, \frac{\delta}{\delta x_{2}}\right]\right]=2 \frac{\partial}{\partial x_{3}}}
\end{array}
$$

We conclude from Corollary 3.2 that $\mathscr{D}$ is not bracket-generating of step 2 on the whole $R^{3 \mid 1}$. It is bracket-generating of step 3.

Example 3.8. Let $\mathscr{M}=R^{1 \mid 2}$ equiped with local supercoordinates $\left(x, \eta_{1}, \eta_{2}\right)$ and $\mathscr{D}$ be the distribution spanned by $\left\{\frac{\delta}{\delta x}=\frac{\partial}{\partial x}, \frac{\delta}{\delta \eta_{1}}=\right.$ $\left.\frac{\partial}{\partial \eta_{1}}+x \frac{\partial}{\partial \eta_{2}}\right\}$. Then $\left[\frac{\partial}{\partial x}, \frac{\delta}{\delta \eta_{1}}\right]=\frac{\delta}{\delta \eta_{2}}$ and from Corollary 3.3, we see that $\mathscr{D}$ is bracket-generating of step 2 on $R^{1 \mid 2}$.

\section{References}

[1] A. Bejancu, On bracket-generating distributions, Int. Electron. J. Geom. 3 (2010) no. 2, 102 - 107.

[2] O. Goertsches, Riemannian supergeometry, Math. Z., 260 (2008) 557--593.

[3] J. Monterde and J. Munoz-Masque and O. A. Sanchez-Valenzuela, Geometric properties of involutive distributions on graded manifolds, Indag. Mathem., N.S., 8 (1997), 217-246.

[4] S. Vacaru and H. Dehnen, Locally Anisotropic Structures and Nonlinear Connections in Einstein and Gauge Gravity, Gen. Rel. Grav., 35 (2003) $209-250$.

[5] S. I. Vacaru, Superstrings in higher order extensions of Finsler Superspaces, Nucl. Phys. B 494 (1997) no. 3, 590-656.

[6] V. S. Varadarajan, Supersymmetry for mathematicians: an introduction, Courant Lecture Notes Series, New York, 2004.

[7] P. C. West, Introduction to supersymmetry and supergravity, Second Edition, World Scientific Pub Co Inc, 1990.

[8] C. D. Zanet, Generic one-step bracket-generating distributions of rank four, Archivum Mathematicum, 51 (2015), 257 - 264. 